\title{
CONDICIONANTES SOCIOLINGÜÍSTICOS DE LA EXPRESIÓN DEL SUJETO PRONOMINAL EN EL SOCIOLECTO ALTO DEL CORPUS PRESEEA-SEVILLA*
}

\author{
Doina Repede \\ Universidad de Sevilla \\ doinarepede@gmail.com
}

\begin{abstract}
Resumen: Este trabajo se propone analizar la variable presencia / ausencia del pronombre personal sujeto junto a un verbo conjugado en el español hablado de la comunidad urbana de Sevilla. En concreto, intentamos establecer cuáles son los factores internos y externos que determinan el empleo del sujeto pronominal en una muestra de 24 entrevistas semidirigidas correspondientes al sociolecto alto y que forman del corpus PRESEEA en la ciudad. Para ello, seguimos la guía de codificación propuesta por Bentivoglio, Ortiz y Silva-Corvalán (2011) para el proyecto panhispánico PRESEEA. Los resultados muestran que el pronombre sujeto se utiliza un $24,8 \%$, y parece estar condicionado, entre otros, por criterios como persona gramatical, especificidad, ambigüedad de la forma verbal, clase semántica del verbo, correfencialidad, edad, etc.
\end{abstract}

Palabras Clave: sujeto pronominal, sociolecto alto, entrevistas semidirigidas, PRESEEA, Sevilla.

\begin{abstract}
This study aims to analysis the variable presence/absence of the personal pronoun subject with a conjugated verb in Spanish language spoken in the city of Seville. Specifically, we try to stablish which internal or external factors are involved in the pronominal subject use in a sample of 24 semi-controlled interviews corresponding to the high sociolect and taken from the PRESEEA-Seville corpus. For this, we base our analysis on the coding guidelines proposed by Bentivoglio, Ortiz and Silva-Corvalán (2011) for the PRESEEA panhispanic project. The results show that the subject pronoun is used in $24,8 \%$ and it is conditioned by different criteria, such as grammatical person, specificity, ambiguity of the verbal form, semantic class of the verb, co-reference or age.
\end{abstract}

Keywords: pronominal subject, high sociolect, semi-controlled interviews, PRESEEA, Seville.

El presente trabajo, que se enmarca dentro del proyecto Patrones Sociolingüísticos del Español de Sevilla (Ref. FFI2015-68171-C5-3-P), financiado por el Ministerio de Economía y Competitividad de España y por el Fondo Europeo de Desarrollo Regional (MINECO/FEDER, UE), ha sido llevado a cabo durante una estancia de investigación en el Departamento de Lengua Española de la Universidad de Granada. Quiero expresar mi agradecimiento al Dr. Antonio Manjón-Cabeza Cruz por haber discutido conmigo diversas cuestiones aquí presentadas, por sus valiosos consejos y sugerencias. 


\section{INTRODUCCIÓN}

El español, al igual que otras lenguas románicas (italiano, portugués, rumano, etc.), se presenta como una lengua pro-drop, es decir, permite la elipsis del pronombre sujeto desde el punto de vista fonético o escrito en oraciones con un verbo conjugado. En este caso, es la flexión verbal la que recupera, parcial o totalmente, la información ausente: yo canto $\sim$ canto; nosotras llamaremos $\sim$ llamaremos $(\mathrm{RAE}$ y ASALE 2009).

La expresión/ausencia del sujeto pronominal ha sido uno de los fenómenos lingüísticos más profusamente analizados en español y se ha llevado a cabo desde distintas perspectivas, tales como la sintáctica, la semántica y la pragmática. Desde un punto de vista tradicional, se ha intentado explicar la presencia del pronombre sujeto como un recurso para enfatizar o desambiguar el contenido expresado. Así, según el Esbozo (1973: 421), el pronombre de sujeto se presenta como una variante libre y se acepta su uso solo en casos en los que se enfatiza el sujeto (para la redundancia del yo y tú) y cuando se trata de ambigüedad - con los pronombres de tercera persona- ${ }^{-}$. En la misma línea, Fernández Soriano (1999: 235-237) contempla también que la expresión del sujeto en el español estándar se debe a la falta de distinción entre la primera y la tercera persona del singular en ciertos tiempos verbales, o por los casos de contraste.

No obstante, la RAE y ASALE (2009: 2555 y ss.) señalan que el uso de la forma pronominal como sujeto aparece como un mecanismo que cumple una función informativa, esto es, la focal. Además, se indican dos aspectos que condicionan la presencia del pronombre personal sujeto: uno es de naturaleza morfológica y otro de carácter léxico. Con respecto al primero, se ejemplifica la primera y tercera persona del pretérito imperfecto de indicativo, y para el segundo, se menciona como ejemplo el pronombre de cortesía usted, que no presenta carácter contrastivo cuando se forma con los imperativos y que aparece en los casos neutros, no marcados ${ }^{1}$.

Por tanto, dado que la presencia del pronombre sujeto en español no se debe al azar, entre los aspectos de interés que ha suscitado este componente lingüístico consiste en determinar de qué depende su aparición en el proceso lingüístico. Los numerosos trabajos realizados sobre el fenómeno que nos ocupa indican que la aparición del sujeto en español se debe a una serie de parámetros de muy diversa naturaleza, como los que se describen a continuación:

a) la clase semántica del verbo. Estudios como el de Enríquez (1984) o Montes Miró (1986) muestran que los verbos que indican acti-

\footnotetext{
${ }^{1}$ Para más detalles sobre la forma usted, véase también Fernández Soriano (1999: 1233).
} 
vidad mental y la postura o el punto de vista del hablante frente a una situación, idea, etc. tal como creer, considerar, estar de acuerdo o saber, influyen en la mayor presencia del pronombre personal sujeto ${ }^{2}$.

b) la correferencialidad (Silva-Corvalán 1982, 1994): cuando el sujeto que se analiza cumple la misma función que en la oración anterior o aparece con otro papel, por ejemplo, de objeto directo. Este criterio "rests on the idea that it will be more probable to mention the subject when it has had a change in the referent, i.e., in order to establish the new topic" (Lastra y Martín Butragueño 2015: 45).

c) la ambigüedad morfológica y fonética. El uso del sujeto pronombre puede ser propiciado, por una parte, por la ambigüedad morfológica entre la primera y la tercera persona en tiempos como el imperfecto y el condicional (Bentivoglio 1987: 41; Silva-Corvalán 1982) y, por otra parte, por la elisión de /-s/ en la conjugación de la segunda persona singular de los verbos en determinadas áreas del español hablado (la denominada "Hipótesis de Compensación Funcional" ${ }^{3}$ de Hochberg 1986).

d) la (in)especificidad del referente (Cameron 1993, 1996) puede determinar la presencia de la forma pronominal sujeto. Se trata de "la situación de especificidad en la que se señala la existencia de un sujeto determinado como el hablante o el oyente, o la situación de no-especificidad en la que se hace referencia a un sujeto indeterminado, general, que puede incluir al hablante, al oyente, o a ninguno de los dos" (Hurtado 2001:16).

Aunque el uso variable del sujeto pronominal se ha ido estudiando aproximadamente desde los años ochenta, aún es difícil hacer generalizaciones al respecto, ya que algunos trabajos no han utilizado la misma metodología ni los mismos criterios de clasificación (Silva-Corvalán 1997) o se basan en "diversos y no siempre adecuados corpora” (Manjón-Cabeza et. al. 2016: 185). Mencionamos, así, entre otros,

\footnotetext{
${ }^{2}$ En un estudio sobre el material recogido por el Instituto Cervantes en 1981, Davidson (1996) evidencia en el español hablado en la ciudad de Madrid un aumento en el uso de pronombres de primera persona con verbos de creencia y conocimiento, verbos que señalan la postura del hablante con respecto a lo que dice. Sobre el mismo aspecto, véase también el trabajo de Miyajima (2000).

${ }^{3}$ Este planteamiento de la hipótesis funcional está basado en la Condición de Distintividad (Distinctivenes Condition) de Kiparsky (1982: 87) que establece que la información semántica relevante se retiene en la estructura superficial del enunciado.
} 
el trabajo de Barrenechea y Alonso (1977) para el español hablado en Buenos Aires, Bentivoglio (1980) sobre el español de Venezuela, Miró y Pineda (1990) sobre el español de la ciudad de Sevilla ${ }^{4}$; y más recientes las investigaciones de Orozco y Guy (2008), Erker y Guy (2012), Shin y Otheguy (2013), Carvalho, Orozco y Shin (2015), Silva-Corvalán (2015) o Peškova (2015).

No obstante, últimamente se están llevando a cabo estudios sobre este aspecto lingüístico que se basan en un corpus homologable con el nuestro, y que forman parte de PRESEEA (Proyecto para el estudio del español de España y América). Así, para el español peninsular, se han centrado en la variabilidad del sujeto pronombre Manjón-Cabeza et. al. (2016) sobre el español hablado en Granada. Para el español de América, mencionamos las investigaciones de Lastra y Martín Butragueño (2015) en México DF, Guerrero (2019) sobre el español hablado en Santiago de Chile u Orozco y Hurtado (2019) en la ciudad de Medellín (Colombia).

Por tanto, el propósito de este trabajo consiste en describir el uso de la presencia/ausencia del pronombre sujeto en el español hablado en la comunidad urbana de Sevilla. En concreto, pretendemos averiguar cuáles son los factores lingüísticos y sociales que condicionan la presencia del sujeto pronominal en la ciudad hispalense. Asimismo, este trabajo resulta pertinente porque, basándonos en las pautas del proyecto panhispánico PRESEEA y en una guía de codificación unificada, nos permite contrastar los resultados obtenidos en la ciudad de Sevilla con los de distintas variedades del español hablado.

\section{METODOLOGÍA Y CORPUS}

\subsection{El corpus}

Para llevar a cabo esta investigación, nos centramos en los materiales del Corpus Sociolingüístico de Sevilla 2009-2018, o PRESEEA-SE, que consiste en entrevistas semidirigidas realizadas a 24 hablantes del sociolecto alto, esto es, con estudios

${ }^{4}$ Este estudio analiza la presencia/ausencia del sujeto pronominal en relación con una serie de variables: 1) textos orales/textos escritos; 2) formas verbales equívocas/no equívocas; 3) tipos oracionales (afirmativas/negativas y principales/subordinadas/subordinadas independientes); 4) grupo generacional de hablantes; 5) sexo de los hablantes (hombres y mujeres). Los autores afirman que, de las variables tenidas en cuenta, solo han resultado significativos los condicionantes sociales. Además, subrayan que cualquier otro factor, "como pueda ser el tipo de oración producida o el carácter ambiguo de las formas verbales empleadas actuarán de forma más débil sobre la probabilidad de aparición del pronombre personal sujeto" (Miró y Pineda 1990: 44). 
universitarios finalizados, máster y/o doctorado 5 . Los hablantes que conforman la muestra empleada para este estudio están estratificados en cuotas de afijación uniforme según edad y sexo (Repede 2019), como se indica en la Tabla 1:

\begin{tabular}{|c|c|c|c|c|}
\hline \multirow{2}{*}{ Sexo } & \multicolumn{4}{|c|}{ Edad } \\
\hline & 20-34 años & 35-54 años & $>55$ años & TOTAL \\
\hline Hombres & 4 & 4 & 4 & 12 \\
\hline Mujeres & 4 & 4 & 4 & 12 \\
\hline TOTAL & 8 & 8 & 8 & 24 \\
\hline
\end{tabular}

Tabla 1: Distribución por cuotas de los informantes de la muestra

\subsection{Selección de casos}

De cada entrevista que forma parte de los materiales manejados en este estudio hemos seleccionado cinco minutos, los comprendidos entre el minuto 5 y el minuto 10. Una vez recogidos los ejemplos, hemos descartado los casos cuando:

a) el pronombre sujeto aparece junto a un verbo en forma no finita:

1. el sótano pues<alargamiento/ $>$ / hay una mesa de pin pong para jugar / los niños y jugar yo también al pin pong (SEVI_H23_055) ${ }^{6}$.

b) el sujeto es indiscutiblemente necesario por ser foco de contraste:

2. I: no tengo<alargamiento/> otra cosa / a lo mejor otro trabajo / aparte / entonces en casa / por las mañanas es que me < vacilación/> aparte es que a mí me gusta // porque no es decir <cita $>$ tú haces esto / yo hago lo otro $</$ cita $>$ si es que si yo echo las < vacilación/ > las mañanas en casa / y es que lo hago todo (SEVI_M13_061).

c) se trata de frases idiomáticas, de tipo yo qué sé, tú sabes, etc.:

\footnotetext{
${ }^{5}$ Véase también http://preseea.linguas.net/Portals/0/Metodologia/METODOLOG\%C3\%8DA\%20PRESEEA.pdf.

${ }^{6} \mathrm{El}$ código del final de los ejemplos debe leerse de la siguiente forma: i) SEVI=Sevilla; ii) sexo: $\mathrm{H}=$ hombre, $\mathrm{M}=$ mujer; iii) edad: $1=20$ a 34 años, $2=35$ a 54 años, $3=55$ años en adelante; iv) grado de instrucción: 3 = estudios superiores; $v$ ) identificación del hablante dentro del corpus: 049, 050, 051 , etc. Sobre las convenciones de transcripción de las entrevistas, véase Repede (2019).
} 
3. <ruido = "chasquido_boca" /> allí en Rubén Darío también / siempre había alguno que fallecía / y aquí también un poco lo mismo / gente muy mayor muy mayor / pero allí por lo menos habían<alargamiento/ > < ruido = "chasquido_boca" / > / yo qué sé / gente<alargamiento/> a lo mejor de cuarenta y cinco cincuenta años (SEVI_M23_066).

d) al pronombre sujeto le sigue una palabra ininteligible o se deja la secuencia incompleta:

4. yo con mi equipaje por ejemplo lo llevaba un<alargamiento/ $>$ burro / que yo no $<$ vacilación/> por medio de la montaña / yo so <palabra_cortada/ $>$ pues he nacido en la ciudad / he crecido en la ciudad y < vacilación/ > / y no <alargamiento/ $>$ soy mmm campestre (SEVI_M13_062).

e) se trata de una repetición del mismo fenómeno. En este caso, se ha computado el mecanismo una sola vez:

5. y claro / ahora el barrio te lo ves que hay un centro comercial / que $<$ alargamiento/ $>$ que hay < vacilación/ $>$ hay por lo menos tres supermercados en la misma calle / eh<alargamiento/> o sea / ya si <vacilación/> veo que ha cambiado / veo que ha cambiado(SEVI_H13_049).

f) cláusulas relativas con sujeto relativizado

6. me encanta la Semana Santa // también también / este año he tenido de todo / porque este año me han invitado a palco / me han invitado a silla / he $<$ alargamiento/ $>$ visto salir algunas procesiones / he visto recoger otra / eh $<$ alargamiento/ $>$ he ido a buscar otras / he tenido balcones que me han invitado amigos también que han tenido balcones en la calle Trajano y también he visto unas pocas (SEVI_H23_056).

También se han eliminado los casos en los que no es posible la presencia de un pronombre personal como los verbos impersonales y las cláusulas con se impersonal. Se han obtenido, así, 879 casos en los que es posible la alternancia entre presencia y ausencia del sujeto pronominal ${ }^{7}$, tal como se refleja en el siguiente fragmento:

${ }^{7}$ Cabe mencionar aquí que en los cinco minutos seleccionados para este análisis no se ha localizado ninguna forma de vosotros o ustedes. Además, no se han incluido en este recuento los 6 ejemplos de uno y una, ya que, en la muestra que hemos manejado para nuestro estudio, no hemos documentado casos de variación. 
7. sí / (Ø) creo recordar / el último verano que $(\varnothing)$ estuve / fue el año que (Ø) hice el preuniversitario / que fue el sesenta y / siete / sí / un poco más de lo que (Ø) te he dicho / o sea que sería del cincuenta y cinco al sesenta y $s<$ alargamiento/ > eis / porque el verano del sesenta y siete $(\varnothing)$ ya no fui a Chipiona / $(\varnothing)$ fui a Cádiz (SEVI_H33_058).

\subsection{Criterios de análisis y procesamiento}

Para codificar los ejemplos recopilados de nuestros materiales hemos seguido principalmente la guía de codificación propuesta por Bentivoglio, Ortiz y Silva-Corvalán (2011) para el proyecto PRESEEA ${ }^{8}$. Por tanto, hemos considerado como variable dependiente la presencia y la ausencia del sujeto pronombre, y como variables independientes las siguientes:

Variables lingüisticas: 2. Persona del sujeto, 3. Especificidad del sujeto, 4. Modo, 5. Tiempo verbal, 6. Progresividad, 7. Perfectividad, 8. Pronominalidad, 9. Valencia verbal, 10. Perífrasis verbal, 11. Ambigüedad de la forma verbal, 12. Clase semántica del verbo, 13. Tipo de cláusula, 14. Correferencialidad, 15. Sujeto previo, 16. Turno de habla, 17. Secuencia discursiva.

Variables sociales: 18. Edad, 19. Sexo.

Con la finalidad de poder establecer estadísticamente si hay relación o no entre las variables dependientes - presencia/ausencia del sujeto pronominal - y las variables explicativas tomadas en consideración en este estudio, hemos aplicado la prueba del ji cuadrado $\left(\chi^{2}\right)$. Si los resultados obtenidos no superan el límite de 0,05 , son estadísticamente significativos e indican que se da una asociación entre las variables nominales. Asimismo, para medir esta relación, empleamos el coeficiente V de Cramer que toma valores entre 0 y 1 . En este caso, los valores cercanos a 0 indican escasa asociación entre las variables y los valores próximos a 1, fuerte asociación.

${ }^{8}$ Sobre los distintos criterios de análisis, véase también Lastra y Martín Butragueño (2015), Manjón-Cabeza et al. (2016), etc. 


\section{ANÁLISIS Y RESULTADOS}

En las 24 entrevistas semidirigidas del sociolecto alto que constituyen la muestra empleada para este estudio y que forman parte del corpus oral PRESEEA-Sevilla, se ha localizado un total de 879 casos de formas de pronombre sujeto, repartidos de la siguiente manera:

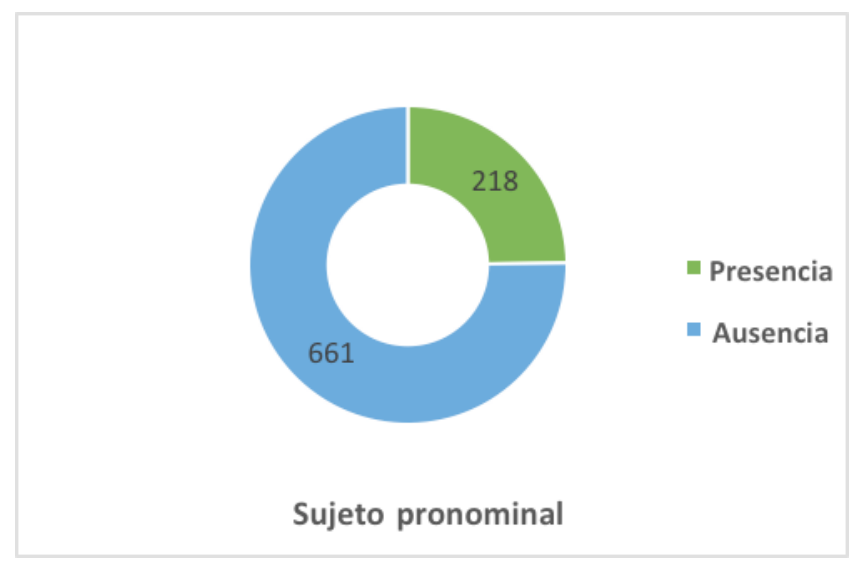

Gráfico 1: Distribución de presencialausencia del sujeto pronominal

Notamos, según el Gráfico 1, que del total de ejemplos de sujetos $(\mathrm{N}=879)$, 218 casos $(24,8 \%)$ aparecen con el pronombre y 661 ejemplos $(75,2 \%)$ sin la forma pronominal. Dado que "Null SPPs are always the predominant solution under any circumstance" (Lastra y Martín Butragueño 2015: 11), intentamos en este estudio averiguar cuáles son los condicionantes internos y externos que propician la presencia del sujeto pronombre.

Tras aplicar la prueba del $\chi^{2}$, observamos que, de las 18 variables consideradas para este estudio, 11 han dado resultados significativos:

\begin{tabular}{|l|l|l|l|l|l|}
\hline \multicolumn{1}{|c|}{ Variables } & \multicolumn{1}{c|}{$\boldsymbol{\chi}^{\mathbf{2}}$} & \multicolumn{1}{c|}{ Sig. } & \multicolumn{1}{c|}{ Variables } & \multicolumn{1}{c|}{$\chi^{\mathbf{2}}$} & \multicolumn{1}{c|}{ Sig. } \\
\hline Persona & $\mathbf{3 5 , 5 4}$ & $\mathbf{0 0 0}$ & Ambigüedad fonética y morfológica & $\mathbf{1 6 , 6 4}$ & $\mathbf{0 0 0}$ \\
\hline Especificidad & $\mathbf{1 5 , 2 9}$ & $\mathbf{0 0 0}$ & Clase semántica del verbo & 64,23 &, 000 \\
\hline Modo verbal & 0,05 &, 823 & Tipo de cláusula & 3,78 &, 151 \\
\hline Tiempo verbal & $\mathbf{2 7 , 5 1}$ & $\mathbf{0 0 0}$ & Correferencia & $\mathbf{3 6 , 0 3}$ & $\mathbf{0 0 0}$ \\
\hline Progresividad & 0,16 &, 069 & Sujeto previo & 3,57 &, 058 \\
\hline
\end{tabular}


Doina Repede

\begin{tabular}{|l|l|l|l|c|c|}
\hline \multicolumn{1}{|c|}{ Variables } & \multicolumn{1}{c|}{$\chi^{\mathbf{2}}$} & \multicolumn{1}{c|}{ Sig. } & \multicolumn{1}{c|}{ Variables } & \multicolumn{1}{c|}{$\chi^{\mathbf{2}}$} & \multicolumn{1}{c|}{ Sig. } \\
\hline Perfectividad & 3,36 &, 067 & Turno de habla & $\mathbf{1 4 , 6 4}$ & $\mathbf{0 0 0}$ \\
\hline Pronominalidad & 3,66 &, 056 & Secuencia discursiva & $\mathbf{1 6 , 4 6}$ & $\mathbf{0 0 0}$ \\
\hline Valencia verbal & 3,58 &, 167 & Edad & $\mathbf{1 2 , 0 5}$ & $\mathbf{, 0 0 0}$ \\
\hline Perifrasis verbal & $\mathbf{1 2 , 1 6}$ &, 002 & Sexo & $\mathbf{4 , 9 6}$ & $\mathbf{, 0 2 6}$ \\
\hline
\end{tabular}

Tabla 2: Significación de variables en la expresión del sujeto pronominal

A la vista de los resultados presentados en la Tabla 2, podemos afirmar que la presencia o ausencia del sujeto está condicionada por diversos criterios internos y externos que vamos a detallar a continuación.

\subsection{Condicionantes lingüísticos y presencia/ausencia del sujeto pronominal}

\subsubsection{Persona gramatical}

El primer factor determinante en la expresión del sujeto es la persona gramati$\mathrm{cal}^{9}$. La primera persona es la más proclive a aparecer, a diferencia de los pronombres plurales que tienden a ser implícitos. Así, del total de 218 casos de pronombres sujetos presentes, se ha localizado un $77,98 \%$ de primera persona, $10,1 \%$ de segunda persona y un 6,42\% de tercera persona singular. En cuanto a la primera y tercera persona de plural, hemos encontrado un $3,7 \%$ y un $1,4 \%$ respectivamente. Si desglosamos por tipo de sujeto pronominal, esto es, presente o ausente, en la Tabla 3 ,

\begin{tabular}{|c|c|c|c|c|c|c|c|}
\hline Variable & Variantes & Presencia & $\%$ & Ausencia & $\%$ & Total & $\%$ \\
\hline \multirow{5}{*}{ Persona } & $1 \mathrm{~s}$ & 170 & 29,62 & 404 & 70,38 & 574 & 65,3 \\
\hline & $2 s$ & 22 & 23,66 & 71 & 76,34 & 93 & 10,6 \\
\hline & $3 s$ & 15 & 28,85 & 37 & 71,15 & 52 & 5,92 \\
\hline & $1 \mathrm{p}$ & 8 & 6,02 & 125 & 93,98 & 133 & 15,13 \\
\hline & $3 p$ & 3 & 11,11 & 24 & 88,89 & 27 & 3,1 \\
\hline Total & & 218 & 24,8 & 661 & 75,2 & 879 & 100 \\
\hline \multicolumn{8}{|c|}{$\chi^{2}=35,54 \quad(4$ g.d.l. $) \quad p=0,000$} \\
\hline
\end{tabular}

Tabla 3: Persona gramatical y presencia/ausencia del sujeto

${ }^{9}$ Nuestros datos están corroborados por estudios previos sobre distintas variedades del español y que han tenido en cuenta este factor lingüístico. Véase, entre otros, el trabajo de Manjón-Cruz et al. (2016) sobre el español de Granada o el de Lastra y Martín Butragueño (2015) en el español hablado en México DF. 
observamos que también son las formas de singular las que más favorecen la presencia del sujeto pronombre, en detrimento de los sujetos plurales, con predominio de la primera persona singular. Así, esta última aparece un 29,62\%, seguida por la tercera $(28,85 \%)$ y segunda persona $(23,66 \%)$. En cuanto a las formas plurales, hemos documentado un $11,11 \%$ para la tercera y un $6,02 \%$ para la primera persona plural. En este sentido, Cameron (1993: 328) afirma que "If we conceive of plural subjects as sets, we find that discourse is typically structured so that the great majority of plural subjects occur in contexts where their set members are either explicitly or inferably present within the immediately preceding discourse". Este tipo de contexto no propicia la aparición de sujetos pronominales, de modo que los sujetos plurales son frecuentemente sujetos nulos (ibídem).

\subsubsection{Especificidad}

El segundo criterio que ha resultado significativo para explicar la presencia/ ausencia del sujeto pronominal es la especificidad. Se afirma que "un argumento es específico cuando hace referencia a un ser, real o imaginario, que se considera identificable, al menos para el emisor. En caso contrario se dice que es inespecífico" (RAE y ASALE 2009: 1134).

De acuerdo con los resultados que se desprenden de la Tabla 4, parece que el sujeto pronominal está condicionado por el rasgo específico del argumento. En este caso, se han documentado 195 ejemplos con sujeto explícito, lo que supone un $27,62 \%$.

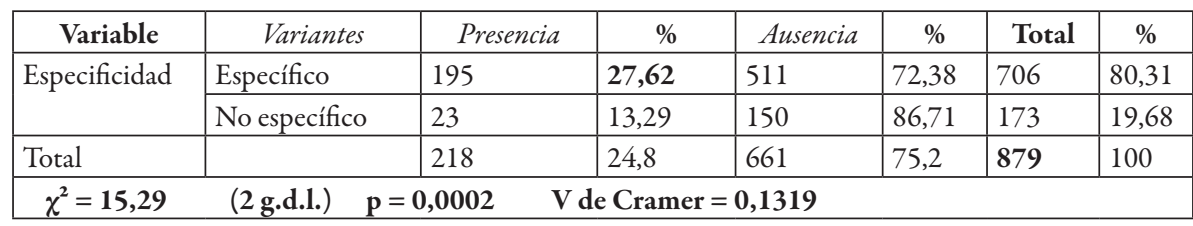

Tabla 4: Especificidad y presencialausencia del sujeto

En cambio, formas no específicas como las que se observan en el fragmento $/ 8 /$,

8. entonces eh<alargamiento/ $>$ en las pasarelas sacarán muchos trajes de manga larga pero no viven la Feria / < simultáneo> día a día </simultáneo> (SEVI_M33_071). 
son menos proclives a la presencia de la forma pronominal sujeto. Se ha documentado, así, un 13,29\% para esta variante.

\subsubsection{Tiempo verbal}

El tiempo verbal es otro factor importante en la presencia de las formas pronominales de sujeto. En esta ocasión, hemos agrupado la variable en presente, pretérito, imperfecto. Dada la escasez de datos para algunos tiempos verbales (futuro ${ }^{10}$, condicional, etc.), hemos considerado incluirlos en la variante otros. Así, de acuerdo con los datos que se presentan en la Tabla 5 ,

\begin{tabular}{|c|c|c|c|c|c|c|c|}
\hline Variable & Variantes & Presencia & $\%$ & Ausencia & $\%$ & Total & $\%$ \\
\hline \multirow{4}{*}{$\begin{array}{c}\text { Tiempo } \\
\text { verbal }\end{array}$} & Presente & 151 & 26,17 & 426 & 73,83 & 577 & 65,64 \\
\hline & Pretérito & 21 & 12,35 & 149 & 87,65 & 170 & 19,34 \\
\hline & Imperfecto & 43 & 38,74 & 68 & 61,26 & 111 & 12,63 \\
\hline & Otros & 3 & 14,29 & 18 & 85,71 & 21 & 2,3 \\
\hline \multirow[t]{2}{*}{ Total } & \multirow{2}{*}{\multicolumn{3}{|c|}{$\begin{array}{c}24,8 \\
p=0,0001\end{array}$}} & 661 & 75,2 & 879 & 100 \\
\hline & & & & \multicolumn{4}{|c|}{ V de Cramer $=0,1769$} \\
\hline
\end{tabular}

Tabla 5: Tiempo verbal y presencia/ausencia del sujeto

el factor más importante en la aparición del sujeto pronominal es el imperfecto con un 38,74\%. Asimismo, cabe mencionar que del total de casos de sujetos con imperfecto $(\mathrm{N}=111), 73(65,77 \%)$ son formas ambiguas, y, de estas, más de la mitad aparecen con sujetos explícitos $(41 / 56,2 \%)$, de modo que "it could suggest a partial functional effect based on the ambiguity constraint" (Lastra y Martín Butragueño 2015: 56). Asimismo, en algunos ejemplos hemos localizado una aglutinación de sujetos pronombres con imperfecto:

9. lo primero que hice fue coger el teléfono / y < vacilación/> y llamé a mi madre / pero simplemente para escuchar su voz / ya ella fue un grito / porque ella sabia que $<$ vacilación/> que ese día me < vacilación/> yo la iba a llamar / cuando llegase a Humahuaca / y ya fue como <cita $>$ yo solamente quería escucharte $</$ cita $>$ / digo $<$ cita > y yo a ti </cita> (SEVI_M13_062).

En cambio, según los resultados anteriores, podemos observar que el pretérito es el tiempo que menos favorece la presencia de esta forma pronominal: hemos lo-

${ }^{10}$ En el corpus manejado, hemos localizado un solo ejemplo de futuro perifrástico con sujeto explícito. En cambio, no se ha documentado ningún caso con futuro morfológico. 
calizado un $12,35 \%$ en los materiales manejados. En lo que toca al resto de tiempos verbales, estos tampoco propician la aparición del pronombre sujeto: se han documentado tan solo 3 ejemplos con sujeto explícito ${ }^{11}$.

\subsubsection{Perifrasis verbal}

También la variable perífrasis verbal ha dado resultados significativos en nuestro estudio. Se ha distinguido, así, entre forma no perifrástica, perífrasis modal (tener + inf., poder + inf.) y perífrasis aspectual (ir a + inf., estar + gerundio $)$. Como se puede comprobar en la Tabla 6,

\begin{tabular}{|c|c|c|c|c|c|c|c|}
\hline Variable & Variantes & Presencia & $\%$ & Ausencia & $\%$ & Total & $\%$ \\
\hline \multirow{3}{*}{$\begin{array}{c}\text { Perífrasis } \\
\text { verbal }\end{array}$} & Forma no perifr. & 207 & 26,64 & 570 & 73,4 & 777 & 88,4 \\
\hline & Modal & 5 & 10,42 & 43 & 89,6 & 48 & 5,5 \\
\hline & Aspectual & 6 & 11,11 & 48 & 88,9 & 54 & 6,14 \\
\hline Total & & 218 & 24,8 & 661 & 75,2 & 879 & 100 \\
\hline \multicolumn{2}{|c|}{$\gamma^{2}=12,16$} & $p=0,00$ & & $V$ de Crame & $=0,1$ & & \\
\hline
\end{tabular}

Tabla 6: Perifrasis y presencialausencia del sujeto

notamos una tendencia a que las formas no perifrásticas favorezcan la presencia del sujeto $(26,64 \%)$ frente a las perifrásticas que parecen propiciar la ausencia de la forma pronominal en función de sujeto: se ha documentado un 89,6\% para las perífrasis modales y un $88,9 \%$ para las aspectuales, esto es, con una diferencia de 0,7 puntos porcentuales entre las dos variantes.

\subsubsection{Ambigüedad}

Otro factor lingüístico que influye en la presencia del pronombre personal sujeto es la ambigüedad ${ }^{12}$. Esta variable se desglosa en dos variantes:

${ }^{11}$ Esta distribución coincide con la de Lastra y Martín Butragueño (2015: 56) sobre el español hablado en México DF: "The most favoring factor is the Co-preterite ( $\mathrm{p}=.60,31.4 \%, 153 / 488$ ); Present is indifferent $(\mathrm{p}=.50,21.1 \%, 187 / 888)$; and Preterite does not favor over SPPs $(\mathrm{p}=.44,15.5 \%$, $86 / 556)$. The rest of verbal tenses have been grouped as "other tenses" and are disfavoring $(.40,15.7 \%$, 17/108)".

${ }^{12}$ Sobre este condicionante lingüístico, véase también, entre otros, el trabajo de Silva-Corvalán (1982) en el español de Estados Unidos o los de Bentivoglio (1980 y 1987) para el español de Venezuela. 
(a) Ambigüedad morfológica y fonética. Se centra, por un lado, en las formas que las gramáticas consideran ambiguas entre $1^{\text {a }}$ y $3^{\text {a }}$ persona del singular, como el condicional y el pretérito imperfecto y pluscuamperfecto de indicativo y subjuntivo (Silva-Corvalán 1982).

10. es que una niña que no sepa bailar sevillanas en Sevilla eso es como<alargamiento/> / como un pecado / <risas = "todos" $/>$ entonces / mi madre / como madre de sevillana / pues me llevaba a una academia a bailar / además daban clases en el mismo colegio / y una vez que se fue la señorita yo quería seguir bailando / o sea / yo lo tenía muy claro que yo quería seguir bailando / y seguí bailando / pero claro / cuando yo ya despuntaba / y ya yo mis padres veían que yo quería seguir en el baile ya como que me cortaba un poquito / la cosa es / bailar sevillanas / pero ya está / porque entonces el mundo del artisteo no era como ahora / no es que yo sea muy mayor / pero las cosas han cambiado mucho en muy poco tiempo (SEVI_M23_067).

Y, por otro, se centra la ambigüedad derivada de la elisión de /-s/ y que se da entre la $2^{\mathrm{a}}$ y $3^{\mathrm{a}}$ persona del singular (tú canta(s) y él canta). Como ya se ha mencionado previamente, este tipo de ambigüedad ha dado lugar a la hipótesis funcional (Hochberg 1986), según la cual la presencia del sujeto pronominal será mayor en aquellas variedades del español donde se produce esa pérdida, ya que contribuye a la desambiguación:

11. E: eso sí / para los que venimos de fuera / es bastante difícil meterte en el círculo I: entonces claro / no es igual / a lo mejor tú viene [s] de fuera y nunca vas a vivir lo que yo he podido vivir / por mucha gente que tú conozca[s] (SEVI_M23_067).

b) Sin ambigüedad. Esta variante recoge los casos en los que aparecen formas verbales no ambiguas:

12. E: uhum / ¿y a ti qué te gusta más el verano o el invierno?

I: a mí / tradicionalmente me ha gustado siempre más el verano // ahora como soy vieja / y el calor me fastidia / mmm pienso que el invierno resguarda más / pero no

E: no

I: el verano es mejor / el verano es más sencillo / sobre todo / y defiende más / lo encuentro // eso de / iy la cama! / hacerla en medio segundo / y no ya tiro de la manta / que se me va la colcha / iJesús / por dios! // nada nada / un horror (SEVI_M33_070).

Por tanto, en el caso de Sevilla, hay que estudiar el papel desambiguador en las formas verbales de la primera y tercera persona singular, así como de la tercera y 
segunda persona singular, debido a que en esta ciudad está generalizada la perdida de la/-s/ en posición final ${ }^{13}$.

Si se observa la Tabla 7 , parece confirmarse que las formas ambiguas favorecen la aparición del pronombre sujeto con mayor frecuencia que cuando se emplean formas verbales no ambiguas. Se ha encontrado, así, un 36\% de forma ambiguas tanto fonéticas como morfológicas, frente a $21,5 \%$ de no ambiguas.

\begin{tabular}{|c|c|c|c|c|c|c|c|}
\hline Variable & Variantes & Presencia & $\%$ & Ausencia & $\%$ & Total & $\%$ \\
\hline \multirow[t]{2}{*}{ Ambigüedad } & $\begin{array}{l}\text { Amb. morfológi- } \\
\text { ca y fonética }\end{array}$ & 72 & 36 & 128 & 64 & 200 & 22,75 \\
\hline & No ambiguo & 146 & 21,5 & 533 & 78,5 & 679 & 77,25 \\
\hline Total & & 218 & 24,8 & 661 & 75,2 & 879 & 100 \\
\hline \multicolumn{8}{|c|}{$\chi^{2}=16,64 \quad(1$ g.d.1. $) \quad p=0,0001$} \\
\hline
\end{tabular}

Tabla 7: Ambigüedad y presencialausencia del sujeto

Si nos fijamos solo en la presencia del pronombre sujeto por clase de persona, según el Gráfico 2, observamos que, en las tres personas gramaticales, según sean ambiguas o no, se dan diferencias en la presencia del pronombre personal sujeto. Así, se puede apreciar que, con respecto a la primera persona, se expresa un $26,7 \%$ para resolver la ambigüedad de la morfología del verbo dada por la coincidencia de esta persona y la tercera del singular. Para la tercera persona, se ha localizado un $60 \%$, si bien este porcentaje corresponde a solo 9 ejemplo del total $(\mathrm{N}=14)$.

Sin embargo, lo más llamativo de este gráfico es el alto porcentaje registrado para la forma ambigua de segunda persona: los hablantes de instrucción alta reponen los pronombres sujetos un 90,9\%. Coincidimos, así, con Ropero (2001: 42) que subraya que "la pérdida de la -s final en los verbos, afecta, sobre todo, al sistema pronominal: las terminaciones verbales fonéticamente casi iguales (como sucede en inglés y en francés) favorecen un uso muy frecuente del pronombre sujeto (como tú quiere(s), tú piensa(s) )"14. Podríamos, por tanto, afirmar que, en la ciudad de Sevilla,

${ }^{13}$ La pérdida de la -s implosiva es un rasgo común a todo el territorio andaluz, que se ha extendido por otras áreas de España e Hispanoamérica. Ya apuntaba Alvar (1955: 287) que "conviene anotar que en Sevilla y Málaga, en el siglo II? <sic>, y en Sevilla, en el siglo VII, se documentaba una pérdida de -s de carácter vulgar, y que hoy en esas mismas provincias se practica idéntica pérdida”.

${ }^{14}$ Estos resultados van en contradicción con los de Ranson (1991) que analiza la variable expresión de pronombres de sujeto en el español hablado en Andalucía. La autora documenta un 91\% de elisión de /s/ y demuestra que en este dialecto la función de los pronombres explícitos no sirve para desambiguar la forma verbal. Así, sus resultados indican que el sujeto pronominal se emplea más con formas verbales que no son ambiguas que con las que sí lo son. En este caso, Ranson explica que el uso variado de pronombres se da más bien en el nivel del discurso, esto es, los pronombres de sujeto 


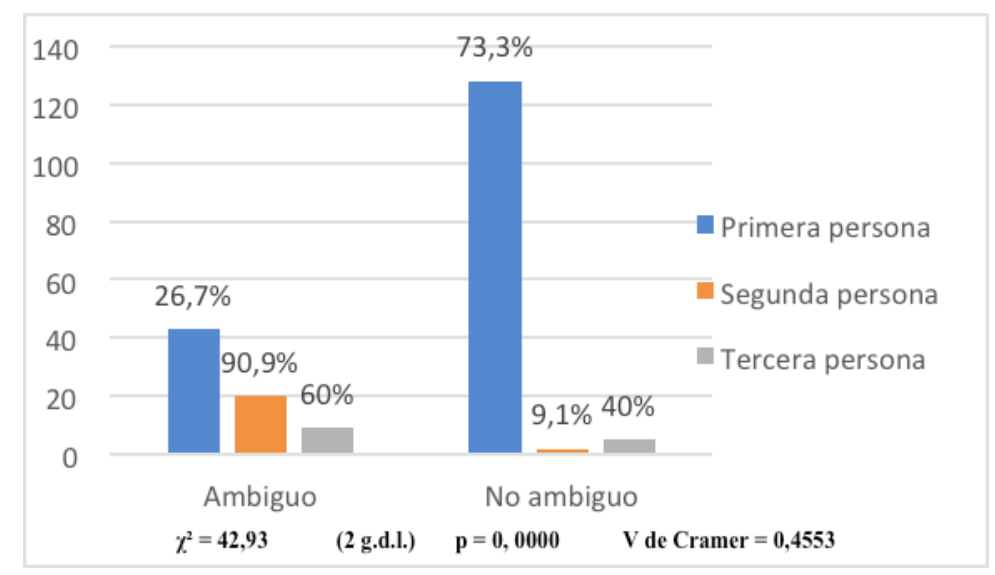

Gráfico 2: Presencia del sujeto y persona gramatical

en el sociolecto alto, se corrobora la idea de que la ambigüedad sí depende del fenómeno de pérdida de la /-s/.

\subsubsection{Clase semántica del verbo}

Un tipo de agrupación que se ha utilizado a menudo con los verbos ha sido la de tener en cuenta únicamente su semántica independientemente del contexto. En este estudio, basándonos principalmente en la guía de codificación de Bentivoglio, Ortiz y Silva-Corvalán (2011) y distintas clasificaciones semánticas de los verbos ${ }^{15}$, hemos optado por crear una variable que permita reunir los verbos en cinco grupos, a saber:

a) entendimiento, que incluye a los de opinión, creencia y procesos mentales (creer, saber, entender). También se han incluido los verbos de percepción sensorial de tipo ver o sentir.

b) actividad (hacer);

c) movimiento (ir, venir, llegar);

d) estado (ser, estar);

aparecen ante la necesidad de indicar el referente cuando contextualmente no está claro: "Because of the role of context in person marking, person can be adequately deduced in the vast majority of the cases even without explicit surface markers. When no contextual markers are present, however, there does appear to be a tendency on the part of speakers to include subject pronouns" (Ranson 1991:149).

${ }^{15}$ Sobre las distintas clasificaciones de verbos según sus rasgos semánticos, véase Bentivoglio (1987), Enríquez (1984), Silva-Corvalán (1994) o Travis (2007). 
e) y dicendi (decir, hablar).

Consideramos, así, que el pronombre personal sujeto tendrá mayor presencia en los verbos del primer grupo frente al resto. Esto se manifiesta en los resultados de la Tabla 8, puesto que la proporción mayor de sujetos explícitos $(44,61 \%)$ se produce con verbos en los que se refleja más el punto de vista y la postura del hablante con respecto a una idea, situación, etc. frente al porcentaje menos favorecedor $(12,12 \%)$ de los verbos de actividad.

\begin{tabular}{|c|c|c|c|c|c|c|c|}
\hline Variable & Variantes & Presencia & $\%$ & Ausencia & $\%$ & Total & $\%$ \\
\hline \multirow{5}{*}{$\begin{array}{l}\text { Clase } \\
\text { semántica }\end{array}$} & Entendimiento & 91 & 44,61 & 113 & 55,39 & 204 & 23,21 \\
\hline & Actividad & 24 & 12,12 & 174 & 87,88 & 198 & 22,53 \\
\hline & Movimiento & 13 & 16,05 & 68 & 83,95 & 81 & 9,22 \\
\hline & Estado & 70 & 21,15 & 256 & 77,34 & 331 & 37,66 \\
\hline & Dicendi & 15 & 23,08 & 50 & 76,92 & 65 & 7,4 \\
\hline Total & & 218 & 24,8 & 661 & 75,2 & 879 & 100 \\
\hline \multicolumn{8}{|c|}{$\chi^{2}=64,23 \quad(4$ g.d.l. $) \quad p=0,0001 \quad V$ de Cramer $=0,2703$} \\
\hline
\end{tabular}

Tabla 8: Clase semántica y presencialausencia del sujeto pronominal

En este caso, nuestros datos coinciden con los ofrecidos en los estudios de Enríquez (1984), Bentivoglio (1987), Blanco (1999) que sostienen que la aparición de los pronombres surge con más frecuencia ante verbos que implican de manera más directa al hablante y a sus opiniones:

13. entonces pues sí que < vacilación/ $>$ tú dices que es<alargamiento/ $>$ menos seria / pero no $<$ vacilación/ $>$ no es menos seria / es igual de seria / lo que pasa que $<$ vacilación/ > que sí / que < vacilación/> que al ser una hermandad de capa no <vacilación/> no está prohibido el poder hablar / eh <alargamiento/> si te tienes que salir a < vacilación/> al cuarto de baño puedes salir / esto en la Catedral / porque / las normas no te dejan salir / en la calle / pero bueno $\mathrm{mmm} /$ yo entiendo que $<$ alargamiento $>$ es una situación / especial / y que < vacilación/ > que hay <alargamiento/> / cada persona es un mundo (SEVI_H13_049).

Si nos centramos en los verbos de entendimiento localizados en nuestro corpus (Gráfico 3), podemos observar que son los verbos creer y recordar los que muestran un porcentaje alto de presencia de sujeto pronominal, esto es, 59,65\% y 61,90\%, respectivamente ${ }^{16}$. Asimismo, todos los casos de $\operatorname{creer}(\mathrm{N}=34)$ y recordar $(\mathrm{N}=13)$

${ }^{16} \mathrm{Al}$ contrario de lo que menciona Rosengren (1974-75: 222) quien indica que son los verbos saber y querer los que presentan la mayor frecuencia de uso pronominal [apud Enríquez (1984: 118)]. 
con sujeto pronominal aparecen en primera persona, de modo que, al enunciar el sujeto de esta persona, el hablante da a conocer como suya una creencia, opinión o suposición. La función del pronombre explícito se mantiene distintiva y contrastiva en cada caso (Luján 1999:1311). Asimismo, "la propia dinámica de la conversación, que pone el foco en la propia experiencia del informante, ayuda a estos datos" (Manjón-Cabeza et al. 2016: 197).

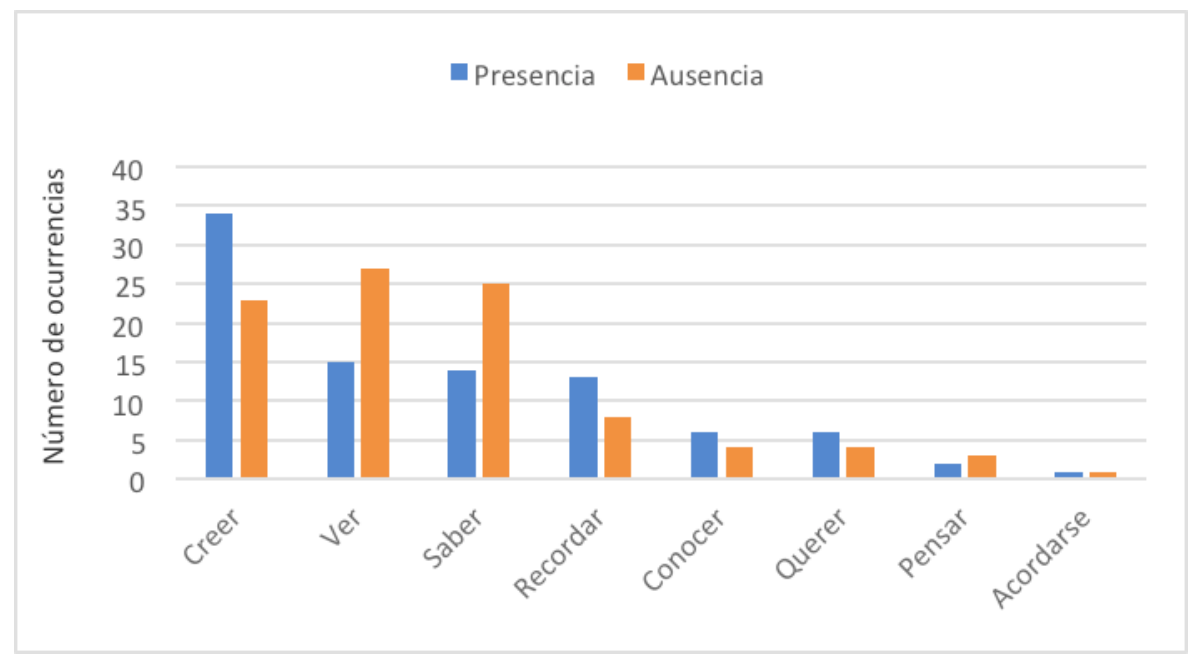

Gráfico 3: Presencia/ausencia del sujeto y verbos de entendimiento

También llama la atención la alta presencia del pronombre sujeto con los verbos conocer y querer (60\% en ambos casos), en detrimento de la ausencia de esta forma, si bien es cierto que se han localizado tan solo 6 ejemplos del total $(\mathrm{N}=10)$ para cada uno de estos verbos.

No obstante, otros estudios sobre la presencia del pronombre sujeto corroboran nuestros resultados: Manjón-Cabeza et al. (2016: 196) también obtienen un porcentaje muy alto de presencia del sujeto pronominal con el verbo creer $(69,12 \%)$ o Aijón y Serrano (2010: 13) que muestran un porcentaje altísimo de presencia ( $75 \%$ del total) de este verbo. 


\subsubsection{Correferencialidad}

Otro criterio importante que ha resultado significativo para explicar la presencia del sujeto pronombre es la correferencialidad. Esta variable se desglosa en tres variantes: correferencial con sujeto previo, como en el ejemplo (14), parcialmente correferencial con cualquier argumento que no sea sujeto (15), no correferencial, esto es, el sujeto no es mencionado en la oración precedente $(16)^{17}$ :

14. mi padre conocía el mundo del artis<palabra_cortada/ > del artista / y Ø sabia los tejemanejes (SEVI_M23_067).

15. lo primero que hice fue coger el teléfono / y <vacilación/> y llamé a mi madre / pero simplemente para escuchar su voz / ya ella fue un grito (SEVI_M13_062).

16. estuve<alargamiento/> en Argentina / en Humahuaca / también me fui<alargamiento $/>$ dos meses / allí fue más < ruido = "chasquido_boca" $/>$ difícil $/$ por las condiciones de vida porque<alargamiento/ $>$ / bueno / estuve < vacilación/ $>$ / estuvimos incomunicados / sin<alargamiento/ $>$ teléfono móvil durante<alargamiento/> / no sé si fue un mes (SEVI_M13_062).

La Tabla 9 recoge los resultados obtenidos con respecto a esta variable:

\begin{tabular}{|c|c|c|c|c|c|c|c|}
\hline Variables & Variantes & Presencia & $\%$ & Ausencia & $\%$ & Total & $\%$ \\
\hline \multirow{3}{*}{ Correferencialidad } & Corr. con sujeto & 24 & 10,4 & 207 & 86,61 & 231 & 26,28 \\
\hline & Corr. con objeto & 31 & 34,44 & 59 & 65,56 & 90 & 10,24 \\
\hline & No correfer. & 163 & 29,21 & 395 & 70,79 & 558 & 63,48 \\
\hline Total & & 218 & 24,8 & 661 & 75,2 & 879 & 100 \\
\hline \multicolumn{8}{|c|}{$\chi^{2}=36,03 \quad$ (2 g.d.1.) $\quad p=0,000 \quad V$ de Cramer $=0,203$} \\
\hline
\end{tabular}

Tabla 9. Correferencialidady presencia/ausencia del sujeto pronominal

Los datos que ofrece el cuadro no solo son estadísticamente significativos, sino que presentan una asociación bastante alta entre las variables $(\mathrm{V}$ de Cramer $=$ $0,203)$. Se desprende, así, que es la correferencia con un argumento diferente al sujeto la que muestra mayor tendencia a la presencia del pronombre $(34,44 \%)$, seguida por la no correferencialidad (29,21\%). Sin embargo, para la correferencia con sujeto se ha documentado solo un $10,4 \%$ de sujetos explícitos, y un altísimo porcentaje de

${ }^{17}$ No se ha tomado en consideración la variante no aplicable, cuando está comenzando un turno de habla, dado que no se ha encontrado ningún ejemplo en los cinco minutos analizados. 
formas verbales con sujeto nulo (86,61\%). Parece, así, que el sujeto no es necesario cuando cumple la misma función sintáctica que el referente, como en el ejemplo $/ 17 /$, donde mi madre es el sujeto explícito de tapaba y también es correferente del sujeto omitido de podía.

17. bueno / mi madre la pobre tapaba cuando podía < risas = "todos"/> / que tú sabes que la autoridad del padre era la autoridad del padre / por lo menos en mi casa (SEVI_M23_067).

\subsubsection{Secuencia textual}

La secuencia textual es un factor que influye también en la presencia del sujeto pronombre en el sociolecto alto de la ciudad de Sevilla. Para esta variable hemos optado por cuatro variantes, a saber: argumentativa + explicativa, narrativa, descriptiva y dialogal. Según los datos incluidos en la Tabla 10,

\begin{tabular}{|c|c|c|c|c|c|c|c|}
\hline Variables & Variantes & Presencia & $\%$ & Ausencia & $\%$ & Total & $\%$ \\
\hline \multirow{4}{*}{ Secuencia textual } & $\begin{array}{l}\text { Argumentativa }+ \\
\text { explicativa }\end{array}$ & 106 & 33,7 & 209 & 66,35 & 315 & 35,84 \\
\hline & Narrativa & 71 & 18,3 & 318 & 81,75 & 389 & 44,25 \\
\hline & Descriptiva & 25 & 25,5 & 73 & 74,49 & 98 & 11,15 \\
\hline & Diálogal & 16 & 20,8 & 61 & 79,22 & 77 & 8,76 \\
\hline Total & & 218 & 24,8 & 661 & 75,2 & 879 & 100 \\
\hline \multicolumn{8}{|c|}{$\chi^{2}=22,89 \quad$ (3 g.d.l.) $\quad p=0,0001$} \\
\hline
\end{tabular}

Tabla 10. Secuencia textual y presencia/ausencia del sujeto pronominal

la argumentación (incluida la explicación) es el factor que más propicia la presencia del sujeto pronominal (33,7\%), "because of the necessity of fixing points of view and underlining one's own and others' opinión” (Lastra y Martín Butragueño 2015: 56), como se muestra en el siguiente fragmento:

18. el verano / yo digo que aquí hay cinco estaciones / el e <vacilación/ > el < vacilación/ > el < vacilación/ > / las cuatro normales y cuando llega / julio y agosto el infierno $<$ risas $=$ "E" $/>/$ yo creo que el verano aquí es mayo y junio y el infierno es julio y agosto / ¿no? / a mí expresamente el <vacilación/> el verano porque lo veo muy desagradable el clima $<$ alargamiento/ $>/$ eh / especialmente julio y agosto / la verdad que co $<$ palabra_cortada/ $>$ con cuarenta y tantos grados no se puede hacer vida normal (SEVI_H23_056). 
También la secuencia descriptiva parece determinar la aparición de las formas pronominales de sujeto en un $25,5 \%$. No obstante, en el polo opuesto se encuentran la circunstancia dialogal y narrativa que son las que menos favorecen la presencia del pronombre sujeto, como en /19/ y /20/:

19. E: uhum / ¿has hecho alguna reforma?

I: no / en breve / espero hacerla

E: ¿qué quieres hacer de reforma?

I: pues<alargamiento/ $>$ / quiero $<$ alargamiento/ $>$ eh $<$ alargamiento/ $>$ / poner la cocina más grande (SEVI_M23_065).

20. I: artístico $/$ o lo que fuera $/ \mathrm{y}$ de hecho $/ \mathrm{mmm} /$ no habitualmente $<$ ruido $=$ "tose I"/> / pero sí de vez en cuando / he encontrado cosas interesantes / cuadros / abanicos / libros / mecheros / relojes / plumas estilográficas // cosas que / a mí me < vacilación/> me divertían / coleccionarlas / y tenerlas / y me gustaban / hoy no / hoy ya voy pues a < vacilación/> a algún libro / alguna cosa puntual que necesite de informática o de<alargamiento/ $>$ herramientas y tal / que se encuentran $<$ vacilación/> y se encuentran con mucha / facilidad además / y además tengo un amigo allí en el Jueves / que es un librero de libros<alargamiento/ > raros antiguos y curiosos / compañero mío de carrera / aunque no llegó a terminarla / pero<alargamiento/> que es / muy buena persona / a mí me quiere muchísimo / y charlo con él / de cosas de < vacilación/ > del mundo / de la actualidad / de política / de cultura / es un hombre muy culto / muy bohemio / muy desastre en su indumentaria / pero / un hombre interesante / $\mathrm{y}<$ alargamiento/ $>$ echo $<$ alargamiento/ $>$ ratos con él / que me < vacilación/ > me entretienen / intercambiamos libros / me regala muchos / y bueno / pues me divierto (SEVI_H33_058).

Así, para las secuencias dialogales, se ha localizado un 20,8\%, y, cuando el hablante relata anécdotas $y / o$ habla de experiencias propias o ajenas, se ha documentado un $18,3 \%$ del total de casos $(\mathrm{N}=389)$.

\subsubsection{Turno de habla}

Como ya se ha mencionado previamente, hemos considerado el criterio turno de habla que se desglosa en dos variantes: mismo turno ${ }^{18}$ y cambio de turno.

${ }^{18}$ No hemos tomado en consideración como interrupciones del turno de habla del informante entrevistado las interjecciones (uhum, ajá, etc.) realizadas por el entrevistador, ya que tales expresiones son un tipo de estímulo, y no se trata de interrupciones o turnos de habla (Bentivoglio 1987). 


\begin{tabular}{|l|l|c|c|c|c|c|c|}
\hline Variables & \multicolumn{1}{|c|}{ Variantes } & Presencia & $\mathbf{\%}$ & Ausencia & $\mathbf{\%}$ & Total & \% \\
\hline \multirow{2}{*}{$\begin{array}{l}\text { Turno de } \\
\text { habla }\end{array}$} & Mismo turno & 170 & 22,48 & 586 & 77,51 & 756 & 86 \\
\cline { 2 - 8 } & Cambio de turno & 48 & $\mathbf{3 9 , 0 2}$ & 75 & 60,98 & 123 & 14 \\
\hline Total & & 218 & 24,8 & 661 & 75,2 & $\mathbf{8 7 9}$ & 100 \\
\hline$\chi^{\mathbf{2}=14,64}$ & (2 g.d.l.) $\quad \mathbf{p}=\mathbf{0 , 0 0 0 1}$ V de Cramer $=\mathbf{0 , 1 3 2 8}$ \\
\hline
\end{tabular}

Tabla 11: Turno de habla y presencia/ausencia del sujeto pronominal

En este caso, parece que el cambio de turno (Tabla 11) favorece la presencia del sujeto pronombre $(39,02 \%)$ en detrimento del mismo turno (22,48\%). Asimismo, lo más usual en este fenómeno es la presencia del sujeto pronombre de primera persona en el cambio de turno, como en $/ 21 /$ :

21.E: y qué te iba a $<$ alargamiento/ $>$ preguntar $\mathrm{R} / \mathrm{a}$ ver $/ \mathrm{y}<$ alargamiento $/>\mathrm{y}$ ¿cuándo? / porque me he enterado de que eras bailaora I: sí yo he bailado siempre / yo lo tenía muy claro desde los seis años E: y cuéntame cómo <alargamiento/ > fue la afición esa del baile o cuándo sentiste tú la llamada del baile o<alargamiento/> I: yo lo tenía clarísimo a los seis años / porque yo < vacilación/> / bueno / en Sevilla todas las niñas las madres las llevaban a las academias para que aprendieran a bailar (SEVI_M23_067).

Coincidimos, así, con Manjón-Cabeza et al. (2016: 204) que afirman que "Sin duda, las reglas lógicas del juego pregunta-respuesta ayudan a lo anterior".

\subsection{Condicionantes sociales y presencia/ausencia del sujeto pronominal}

Los dos factores extralingüísticos sexo y edad considerados en este estudio también han dado resultados significativos. Con respecto al primero, presentamos en la Tabla 12, los datos obtenidos en nuestro análisis:

\begin{tabular}{|c|c|c|c|c|c|c|c|}
\hline Variable & Variantes & Presencia & $\%$ & Ausencia & $\%$ & Total & $\%$ \\
\hline \multirow[t]{2}{*}{ Sexo } & $\mathrm{H}$ & 77 & 20,87 & 292 & 79,13 & 369 & 41,98 \\
\hline & $M$ & 141 & 27,65 & 369 & 72,35 & 510 & 58,02 \\
\hline Total & & 218 & 24,8 & 661 & 75,2 & 879 & 100 \\
\hline$\chi^{2}=4,92$ & (1 g.d.l.) & $=0,0265$ & Cram & $=0,0775$ & & & \\
\hline
\end{tabular}

Tabla 12: Sexo y presencia/ausencia del sujeto pronominal 
Se desprende, así, que son las mujeres las que más uso hacen del sujeto pronominal (27,65\%) frente a los hombres (20,87\%). Cabe mencionar también que del total de pronombres sujeto de primera persona anotados $(\mathrm{N}=170)$, los hombres lo utilizan un $38,82 \%$, mientras que las mujeres casi duplican el empleo de esta forma pronominal, esto es, un $61,18 \%$. Para las demás personas gramaticales, el uso del pronombre sujeto también predomina en las mujeres.

Y, por último, la Tabla 13 recoge los resultados obtenidos para la variable edad $^{19}$.

\begin{tabular}{|c|c|c|c|c|c|c|c|}
\hline Variables & Variantes & Presencia & $\%$ & Ausencia & $\%$ & Total & $\%$ \\
\hline \multirow{3}{*}{ Edad } & $20-34$ & 76 & 27,74 & 198 & 72,26 & 274 & 31,2 \\
\hline & $35-54$ & 80 & 29,74 & 189 & 70,26 & 269 & 30,6 \\
\hline & $55 y+$ & 62 & 18,45 & 274 & 81,56 & 336 & 38,2 \\
\hline Total & & 218 & 24,8 & 661 & 75,2 & 879 & 100 \\
\hline$\chi^{2}=12,05$ & \multicolumn{7}{|c|}{$(2$ g.d.l. $) \quad p=0,0024 \quad V$ de Cramer $=0,1171$} \\
\hline
\end{tabular}

Tabla 13. Edady presencia/ausencia del sujeto pronominal

Podemos observar que es la tercera generación del sociolecto alto la que menos uso hace del sujeto pronombre (18,45\%), seguida del primer grupo etario $(27,74 \%)$. En cambio, los hablantes de la segunda generación son los que más utilizan el pronombre sujeto (29,74\%). Estos datos van en contradicción con los obtenidos en otras variedades del español peninsular (Manjón-Cabeza et al.2015), o del español de Mexico DF (Lastra y Martín-Butragueño 2015), colombiano (Orozco 2015) y dominicano (Alfaraz 2015) que indican que son los informantes de primera generación los que menos reponen los pronombres sujetos.

Al cruzar el factor edad con la persona gramatical, se aprecia en el Gráfico 4 que es la primera generación quien más utiliza el sujeto pronombre de primera persona $^{20}$ (38,8\% frente al $32,4 \%$ de la tercera y $28,8 \%$ de la segunda generación). No obstante, es el segundo grupo etario de instrucción alta quien se caracteriza por las segundas personas con pronombre: se ha localizado, por tanto, un $72,3 \%$ de formas de pronombre sujeto para este grupo de edad.

${ }^{19}$ Los trabajos de Orozco y Guy (2008) para Barranquilla (Colombia) y Manjón-Cabeza et al. (2016) para Granada sobre el fenómeno objeto de nuestro análisis, también indican que la edad es un factor social significativo en la ausencia del sujeto pronominal.

${ }^{20}$ Estos datos nos hacen pensar que las distintas comunidades de habla pueden asignar valores sociales diferentes a la misma variable lingüística y que todo ello podría conducir a resoluciones diferentes de esta variación, un tema digno de futuras investigaciones. 


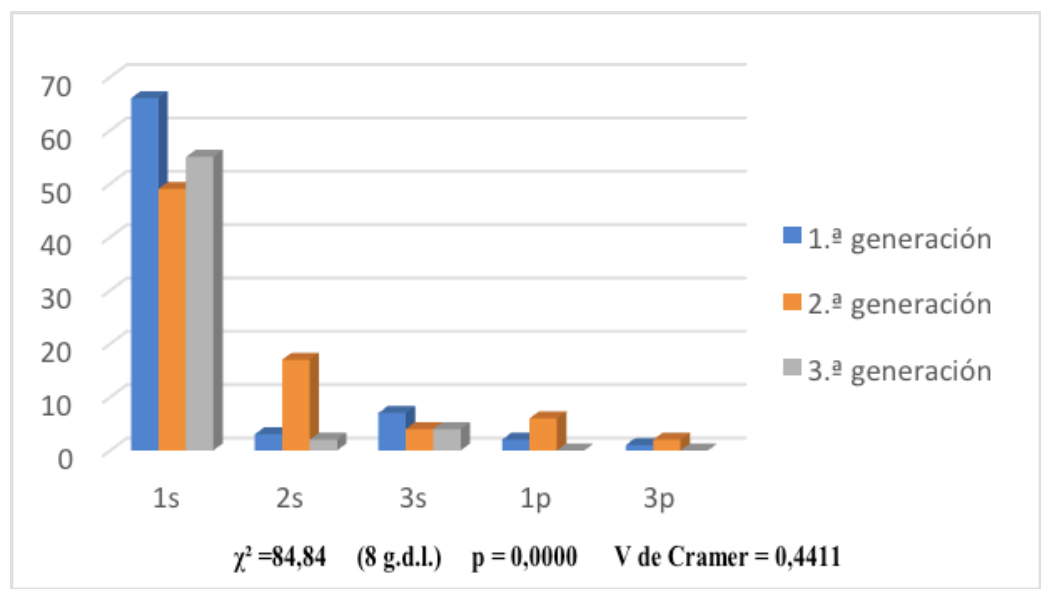

Gráfico 4. Presencia del pronombre sujeto según grupos de edades

\section{CONCLUSIONES}

En las 24 entrevistas semidirigidas del sociolecto alto en la comunidad urbana de Sevilla se ha localizado un $24,8 \%$ de presencia de formas pronominales de sujeto. $\mathrm{Al}$ igual que en otras comunidades de habla hispana (Manjón-Cabeza et al. 2016, Lastra y Martín Butragueño 2015), la aparición o ausencia del sujeto pronominal está condicionada por múltiples factores: persona gramatical, especificidad, tiempo verbal, perífrasis verbal, ambigüedad fonética y morfológica, clase semántica del verbo, correferencialidad, turno de habla, secuencia discursiva, sexo y edad.

Así, la primera persona del singular se caracteriza por un alto porcentaje de presencia (29,62\%), en detrimento de las demás personas. Asimismo, las personas de singular se oponen a las de plural, ya que estás últimas presentan unos porcentajes muy bajos. La especificidad también ayuda a la aparición del sujeto pronominal, pues las expresiones inespecíficas tienden al rechazo del pronombre personal.

Con respecto a la ambigüedad, la coincidencia de las marcas verbales de persona y número gramatical entre la $1^{\mathrm{a}}$ y $3^{\mathrm{a}}$ persona, singular, favorece la aparición del sujeto. No debe descuidarse que este factor va de la mano del imperfecto, ya que las formas ambiguas son típicamente las de primera y tercera persona del singular de este tiempo verbal. Asimismo, en la ciudad de Sevilla, la ambigüedad derivada de la elisión de /-s/ que se da entre la $2^{\mathrm{a}}$ y $3^{\mathrm{a}}$ persona del singular también promueve la aparición del sujeto pronominal. Por tanto, mencionar explícitamente un sujeto 
puede entenderse como una estrategia lingüística que usan los hablantes para desambiguar los contextos que podrían causar ambigüedad.

Relativo a los condicionantes semántico-pragmáticos, como ocurre en otras comunidades de habla hispana, los verbos que implican procesos mentales de tipo creer, recordar, etc. muestran una preferencia por los sujetos explícitos, con predominio de la primera persona del singular en secuencias de tipo argumentativo-explicativas. En lo que toca a la correfencialidad, de nuestro análisis se desprende que existe una tendencia a mencionar el sujeto pronominal cuando antes ha aparecido cumpliendo otro papel sintáctico. El cambio de turno también propicia la presencia del pronombre sujeto, pero se trata de un factor íntimamente ligado a la persona gramatical, dado que es la $1^{\text {a }}$ persona del singular la que predomina en esta posición. En los que concierne los criterios sociales, son las mujeres y la segunda generación quien más utilizan los sujetos explícitos.

Por último, a la vista de los resultados obtenidos en este análisis y para tener una visión más amplia del uso variable del sujeto pronombre en la comunidad de Sevilla, se hace necesario abordar en futuras investigaciones el estudio de los otros dos sociolectos - medio y bajo- ${ }^{-}$para ver qué cuáles son los criterios sociolingüísticos que condicionan su aparición en la ciudad de Sevilla.

\section{BIBLIOGRAFÍA}

Aijón Oliva, Miguel Ángel y María José Serrano (2010): «El hablante y su discurso: expresión y omisión del sujeto de creo», Oralia, 13, pp. 7-38.

Alfaraz, Gabriela G. (2015): "Variation of Overt and Null Subjects Pronouns in the Spanish of Santo Domingo", en Ana M. Carvalho, Rafael Orozco y Naomi Lapidus Shin (eds.), Subject Pronoun Expression in Spanish: A Cross-dialectal perspective, Washington DC, Georgetown University Press, pp. 3-16.

Alvar, Manuel (1955): «Las hablas meridionales de España y su interés para la lingüística comparada », Revista de Filología Española, 39/1-4o pp. 284-313.

Barrenechea, Ana María y Alicia Alonso (1977): «Los pronombres personales sujetos en el español hablado en Buenos Aires», en Juan M. Lope Blanch (ed.), Estudios sobre el español hablado en las principales ciudades de América, México, UNAM, pp. 333-349.

Bentivoglio, Paola (1980): Why canto and not yo canto? The problem offirst-person subject pronoun in spoken Venezuelan Spanish. M. A. Thesis, Los Angeles, University of California. 
Bentivoglio, Paola (1987): Los sujetos pronominales de primera persona en el habla de Caracas. Caracas, Universidad Central de Venezuela.

Bentivoglio, Paola, Luis A. Ortiz y Carmen Silva-Corvalán (2011): La variable expresión del sujeto pronominal. Guía de codificación. Disponible en http://preseea.linguas.net/Portals/0/Metodologia/guia_codificacion_sujetos_julio_2011.pdf. [18/06/19]

Cameron, Richard (1993): «Ambiguous agreement, functional compensation, and non-specific tú in the Spanish of San Juan, Puerto Rico and Madrid, Spain », Language Variation and Change, 5, pp. 305-334.

CAmeron, Richard (1996): «A community-based test of a linguistic hypothesis», Language in Society, 25/1, pp. 61-111.

Carvalho, Ana, Rafael Orozco y Naomi Shin (2015): Subject Pronoun Expression in Spanish: A cross-dialectal perspective, Washington DC, Georgetown University Press.

Davidson, Brad (1996): «'Pragmatic weight' and Spanish subject pronouns: The pragmatic and discourse uses of tú and yo in spoken Madrid Spanish », Journal of Pragmatics 26, pp. 543-565.

ENRÍQUEZ, Emilia V. (1984): El pronombre personal sujeto en la lengua hablada en Madrid, Madrid, Consejo Superior de Investigaciones Científicas.

ERKer, Daniel y Gregory R. GuY (2012): «The role of lexical frequency in syntactic variability: variable subject personal pronoun expression in Spanish», Language, 88, pp. 526-557.

FERnÁndez Soriano, Olga (1999): «El pronombre personal. Formas y distribuciones. Pronombres átonos y tónicos», en Ignacio Bosque y Violeta Demonte (eds.): Gramática Descriptiva de la Lengua Española, Madrid, Espasa Calpe, pp. 1209-1273.

Guerrero, Silvana (2019): «Expresión del sujeto pronominal en el corpus PRESEEA de Santiago de Chile». Ponencia en el Seminario Internacional Las investigaciones lingüisticas en el Mundo Hispánico. Cuba, La Habana, 30 de enero -1 de febrero de 2019.

Hochberg, Judith G. (1986): «Functional compensation for /s/ deletion in Puerto Rican Spanish», Language, 62, pp. 609-621.

Hurtado Cubillos, Luz Marcela (2001): La variable expresión del sujeto en el español de los colombianos y colombo-americanos residentes en el condado Miami-Dade. Tesis doctoral, Universidad de Florida.

KIPARSKY, Paul (1982): Explanation in phonology. Dordrecht, Foris. 
LUJÁN, Marta (1999): «Expresión y omisión del pronombre personal», en Ignacio Bosque y Violeta Demonte (eds.), Gramática descriptiva de la lengua española, vol. 1, Madrid, Espasa Calpe, pp. 1275-1315.

Lastra, Yolanda y Pedro Martín Butragueño (2015): «Subject Pronoun Expression in Oral Mexican Spanish», en Ana M. Carvalho, Rafael Orozco y Naomi Lapidus Shin (eds.), Subject Pronoun Expression in Spanish: $A$ Cross-dialectal perspective, Washington DC, Georgetown University Press, pp. $39-57$.

Majón-Cabeza Cruz, Antonio et al. (2016): «Factores determinantes en la expresión del sujeto pronominal en el corpus PRESEEA Granada», Boletín de Filología, 51/2, pp.181-207.

Miró Vera, Ramona y Miguel Ángel de Pineda Pérez (1990): «Determinación sociolingüística de la presencia/ausencia del pronombre personal sujeto», en M. ${ }^{a}$ Teresa Palet (ed.), Sociolingüistica andaluza 5. Habla de Sevilla y hablas americanas, Sevilla, Universidad de Sevilla, pp. 37-44.

Miyajima, Atsuko (2000): «Aparición del pronombre sujeto en español y semántica del verbo», Sophia Lingüistica, 46-47, pp. 73-88.

Montes Miró, Rosa Graciela (1986): «Los factores discursivos en el análisis de los pronombres personales sujeto en español», Morphé, 2, pp. 45-71.

Orozco, Rafael y Gregory R. GuY (2008): «El uso variable de los pronombres sujetos: ¿qué pasa en la costa Caribe colombiana?», en Westmoreland, Maurice y Juan Antonio Thomas (eds.), Selected Proceedings of the 4th Workshop on Spanish Sociolinguistics, Somerville, MA, Cascadilla Proceedings Project, pp.70-80.

Orozco, Rafael y Luz Marcela Hurtado Cubillos (2019): «A Sociolinguistic Study of Pronominal Expression in Mainland Colombian Spanish», ICLAVE 10, International Conference on Language Conference in Europe. Leeuwarden, 26-28 de junio de 2019.

Otheguy, Ricardo y Ana Celia Zentella (2012): Spanish in New York: Language contact, dialectal leveling, and structural continuity, Oxford, Oxford University Press.

PEŠKovA, Andrea (2015): Sujetos pronominales en el español porteño. Implicaciones pragmáticas en la interfaz sintáctico-fonológica, Berlin, De Gruyter Mouton.

Real Academia Española (1973): Esbozo para una nueva gramática de la lengua española, Madrid, Espasa-Calpe.

Real Academia Española y Asociación de Academias de la Lengua Española (2009): Nueva Gramática de la lengua española, Madrid, Espasa.

RANSON, Diana (1991): «Person marking in the wake of /s/ deletion in Andalusian Spanish », Language Variation and Change, 3/2, pp. 133-152. 
Repede, Doina (ed.) (2019): El español hablado en Sevilla. Corpus PRESEEA-Sevilla. Vol. I Hablantes de instrucción alta, Sevilla, Editorial Universidad de Sevilla.

Ropero, Miguel (2001): «Sociolingüística andaluza: problemas y perspectivas», en Carbonero, Pedro y Rosario Guillén (eds.), Sociolingüistica andaluza, 12. Identidad lingüistica y comportamientos discursivos, Sevilla, Secretariado de Publicaciones de la Universidad de Sevilla, pp. 21-48.

Shin, Naomi Lapidus y Ricardo OTheguy (2013): «Social class and gender impacting change in bilingual settings: Spanish subject pronoun use in New York», Language in Society, 42, pp. 429-452.

Silva-Corvalán, Carmen (1982): «Subject expression and placement in spoken Mexican-American Spanish», en Jon Amastae y Lucía Elías-Olivares (eds.), Spanish in the United States, Cambridge (MA), Cambridge University Press, pp. 93-120.

Silva-Corvalán, Carmen (1994): Language Contact and Change. Spanish in Los Angeles, Oxford, Clarendon Press.

Silva-Corvalán, Carmen (1997): «Variación sintáctica en el discurso oral: problemas metodológicos», en Francisco Moreno Fernández (ed.), Trabajos de sociolingüistica hispánica, Universidad de Alcalá, Nuevo Siglo, pp. 115-135.

Silva-Corvalán, Carmen (2015): «Infantes bilingües y hablantes de herencia adultos: ¿Qué los vincula?», Boletín de Filologia, 50/1, pp. 165-191.

Travis, Catherine (2007): «Genre effects on subject expression in Spanish: Priming in narrative and conversation», Language Variation and Change, 19/2, pp. 101-135.

Fecha de recepción: 17 de julio de 2019

Fecha de aceptación: 25 de septiembre de 2019 
